\title{
Recombinant Human Erythropoietin for Treating Treatment-Resistant Depression: A Double-Blind, Randomized, Placebo-Controlled Phase 2 Trial
}

\author{
Kamilla W Miskowiak*,', Maj Vinberg', Ellen M Christensen', Jens D Bukh', Catherine J Harmer², \\ Hannelore Ehrenreich ${ }^{3}$ and Lars V Kessing' \\ 'Psychiatric Centre Copenhagen, Copenhagen University Hospital, Rigshospitalet, Copenhagen, Denmark; ${ }^{2}$ Department of Psychiatry, University \\ of Oxford, Oxford, UK; ${ }^{3}$ Clinical Neuroscience, Max Planck Institute of Experimental Medicine, Göttingen, Germany
}

\begin{abstract}
Pharmacological treatments for depression have insufficient efficacy in 30-40\% of patients and fail to reverse cognitive deficits. Erythropoietin (EPO) has neurotrophic actions and aids neurocognitive function. The aim of this exploratory study was to determine whether recombinant human EPO improves mood and memory in treatment-resistant depression. Forty treatment-resistant depressed unipolar patients with Hamilton Depression Rating Scale- 17 (HDRS-17) score $\geqslant 17$ were randomized to eight weekly EPO (Eprex; $40000 \mathrm{IU}$ ) or saline infusions in a double-blind, placebo-controlled, parallel-group design. Patients were assessed at baseline and at weeks 5, 9, and 14. Primary outcome was reduction in HDRS- 17 score. Global assessment of function (GAF) was reported in addition. Secondary outcome was remission rate, and tertiary outcomes were changes in Rey Auditory Verbal Learning Test (RAVLT), Beck Depression Inventory-2I (BDI-2I), and World Health Organization Quality of life-BREF (WHOQOL-BREF). Exploratory outcomes were depression and cognition composite scores. HDRS- 17, GAF, and remission rates showed no effects of EPO over saline at week 9 (P-value $\geqslant 0.09)$. However, EPO improved BDI $(P=0.02)$ and WHOQOL-BREF $(P=0.0 \mathrm{I})$, and this was maintained at follow-up week 14 ( $P$-values $\leqslant 0.04)$. EPO enhanced verbal recall $(P=0.02)$ and recognition $(P=0.03)$, which was sustained at follow-up $(P$-values $\leqslant 0.04)$. Exploratory analysis in patients fulfilling depression severity criteria at trial start revealed ameliorated HDRS- I7 in EPO (N=14) vs saline groups $(N=17)$, which was sustained at week 14 (P-values $\leqslant 0.05)$. Exploratory analysis in the complete cohort showed that EPO reduced depression composite at weeks 9 and 14 (P-values $=0.02)$. The findings of this exploratory study highlight EPO as an interesting compound for treatment-resistant depression, which deserves further investigation.

Neuropsychopharmacology (2014) 39, 1399-1408; doi:I0.1038/npp.2013.335; published online 22 January 20I4
\end{abstract}

Keywords: cognitive; depression; erythropoietin; memory; treatment resistant

\section{INTRODUCTION}

Depression is a common, life-disrupting, and highly recurrent disorder and among the leading causes of disability worldwide (Moussavi et al, 2007). Current antidepressant drug strategies have insufficient efficacy in $30-40 \%$ of patients (Rush et al, 2006) and fail to reverse cognitive dysfunction (Hasselbalch et al, 2011). These shortcomings lead to impaired social and occupational function, poor physical health, and significant suicide rates. Progress is thus urgently needed to address treatmentresistant depression and attenuate cognitive deficits. Evidence that restoration of synaptic plasticity may be an important mechanism of chronic antidepressant treatment

\footnotetext{
*Correspondence: Dr KW Miskowiak, Psychiatric Centre Copenhagen, Copenhagen University Hospital, Rigshospitalet, Blegdamsvej 9, 2100 Copenhagen, Denmark, Tel: +45 3864 7087, Fax: + 4538647077 , E-mail: Kamilla@miskowiak.dk

Received 28 August 2013; revised 28 November 2013; accepted 4 December 2013; accepted article preview online 10 December 2013
}

highlights compounds that directly increase cellular resilience and plasticity as novel faster acting treatments (Manji et al, 2003). New drug targets that indirectly affect neuroplasticity through modulation of glutamatergic and cholinergic systems (Furey and Drevets, 2006; Zarate et al, 2006) appear to have rapid antidepressant efficacy in treatment-resistant depression. However, their clinical potential is limited by neurotoxic actions with prolonged exposure and deleterious effects on memory function (Curran and Morgan, 2000; Danysz et al, 1988). There are hence no current or investigational drugs that address the clinical need for improvement of both depressive symptoms and cognitive function.

Erythropoietin (EPO) is well known for its effects on red blood cells but is also produced in the brain (Marti et al, 1996) where it has a key role in neuroprotection and development (Siren et al, 2009), and in cognitive function (Kästner et al, 2012; Sargin et al, 2010). Systemically administered EPO crosses the blood-brain barrier (Brines et al, 2000) and exerts neuroprotective and neurotrophic effects in neuropsychiatric conditions (Siren et al, 2009). 
These neuroadaptive actions are accompanied by enhanced cognitive function in rodent models of acute and chronic neural injury, neurodegenerative conditions, and in healthy rodents, independent of changes in the erythroid cell line (Siren et al, 2009; Sargin et al, 2010). Translational studies have shown beneficial effects of long-term EPO treatment on cognition in neuropsychiatric patients with cognitive decline (Ehrenreich et al, 2007a, b). Various neurobiological mechanisms mediate these actions, including activation of antiapoptotic, antioxidant, and anti-inflammatory signaling in neurons, glial, and cerebrovascular endothelial cells, promotion of dendritic sprouting and upregulation of hippocampal brain-derived neurotrophic factor, and neurogenesis (Brines et al, 2000; Ehrenreich et al, 2007a, b; Sargin et al, 2010).

We have previously demonstrated that a single high dose of EPO improves neurocognitive function in healthy and depressed individuals in the absence of changes in red cell mass (Miskowiak et al, 2012). In particular, EPO enhanced memory-relevant hippocampal response, improved executive function and had a remarkably similar profile of effects to serotonergic antidepressants on the processing of emotional information (Miskowiak et al, 2007a, b, 2008). Consistent with this, recent preclinical studies revealed antidepressant-like effects and memory improvement with EPO treatment, which were mediated by enhanced hippocampal plasticity and long-term potentiation (Adamcio et al, 2008; Girgenti et al, 2009; Leconte et al, 2011). This converging evidence highlights EPO as a candidate therapeutic agent in depression. The present exploratory study therefore aimed to explore whether the early neurocognitive and antidepressant-like effects of single EPO administration translate into improved mood and memory with repeated administration to patients with treatment-resistant depression.

\section{PATIENTS AND METHODS}

\section{Study Design and Participants}

The trial design has been published in full (Miskowiak et al, 2010). The study had a double-blind, placebo-controlled, parallel-group design. We recruited patients through Clinic for Affective Disorders, Department of Psychiatry, Copenhagen University Hospital, Rigshospitalet and by advisement on relevant websites. Patients were screened with Schedules for Clinical Assessment in Neuropsychiatry (SCAN).

Eligible patients had a diagnosis of major depression of moderate severity (Hamilton Depression Rating Scale-17 (HDRS-17) score >17), were aged 18-65 years, and fulfilled the criteria for treatment resistance based on assessment of their medical treatment history with the Treatment Response to Antidepressants Questionnaire (TRAQ) (Posternak et al, 2004). For each antidepressant trial, the duration, dosages, compliance, and outcome were recorded. Adequate antidepressant trial was defined in accordance with the Antidepressant Treatment History Form (ATHF) by Sackeim (2001) as treatment with an antidepressant drug for $>4$ weeks in a sufficient dosage (corresponding to a score of 3 or above in the ATHF) and with $>85 \%$ compliance (corresponding to a score of 4 in the TRAQ). Treatment resistance was established, when remission was not obtained after minimum two adequate antidepressant treatments with two different classes of antidepressant drugs in previous and/ or current mood episodes (which in the present cohort had a mean duration of 4.6 years $(95 \%$ CI: 2.5-6.7 years).

In these patients who had failed adequate treatments with at least two classes of antidepressants, there is no evidence for efficacy of a third type of antidepressant treatment (Rush et al, 2006). Nevertheless, it was an inclusion criterion that patients had made no changes in their antidepressant medication for at least 2 weeks before trial initiation to minimize the risk of potential confounding effects of antidepressant treatment on mood symptoms during the trial. Exclusion criteria were significant medical conditions (diabetes, renal failure, epilepsy, hypertension, present or past malignancies, and thromboses), smoking, BMI $>30 \mathrm{~kg} / \mathrm{m}^{2}$, body weight $<45$ or $>95 \mathrm{~kg}$, bipolar disorder, schizophrenia, alcohol or substance misuse, acute suicidal risk, pregnancy or breast feeding, contraceptive medication or a first-degree family history of thromboembolic events, or seizure disorders. Benzodiazepines were tapered to a maximum of $22.5 \mathrm{mg}$ oxazepam (or equivalent). Pregnancy tests were performed on female patients in their fertile age before and every second week during the study. Blood screening and physical examinations were undertaken at baseline and weekly during the treatment period, and at three follow-up visits to ensure patient safety. Written informed consent was obtained from all patients before their inclusion and letters were sent to their general practitioners to rule out history of significant medical conditions. The procedures were in accordance with the ethical standards of the Danish Research Ethics Committee for the Capital Region, and the study was approved by the committee (H-C-2008-092), Danish Medicines Agency (2612-4020), and Danish Data Agency (2008-41-2711) (the trial is registered in clinicaltrials.gov identifier: NCT00916552.

Using nQuery Advisor 5.0 software, we calculated that a sample size of 40 would reach $86 \%$ power to detect a clinically relevant difference (of three points) in the primary outcome measure (HDRS-17 scores) between the two treatment groups at an $\alpha$ level of 5\% (two-sided test) (Miskowiak et al, 2010).

\section{Randomization and Masking}

Block randomization was performed by Pharma Consulting Group (pharmaconsultinggroup.com) with stratification for age $(<$ or $\geqslant 35$ years $)$ and gender. Patients, study nurses, and outcome assessors were blinded to group assignment and blinding was maintained throughout the study, data management, outcome assessment, and data analysis. The randomization list was kept in a locked filing cabinet to which only the person preparing the study medication had a key. Preparation of study medication was performed to ensure double-blinding at the time of infusion: $1 \mathrm{ml}$ recombinant human EPO (Eprex; 40000 IU; Janssen-Cilag) or saline $(\mathrm{NaCl} 0.9 \%)$ kept at a temperature of $5-8{ }^{\circ} \mathrm{C}$ was injected into a standard $100 \mathrm{ml}$ saline $(\mathrm{NaCl} 0.9 \%)$ infusion bag, which was then given to the study nurse or physician administering the medication. Double-blinding was further ensured by EPO, a colorless liquid indistinguishable from saline. Weekly monitoring of blood tests and any side 
effects was performed by a physician not involved in outcome measure assessments. The good clinical practice unit at Copenhagen University Hospital (http: //www. gcp-enhed.dk/kbh/) monitored that blinding was maintained throughout the study and filed no report on any breach of blinding.

\section{Procedures}

Patients were randomized after the screening session and started the trial 2 weeks later for logistic reasons. They then received weekly infusions of either EPO (40000 IU) or saline for 8 weeks (weeks 1-8) in addition to their current antidepressant medication. This high dose and treatment schedule were chosen because several studies have shown that weekly administration of EPO in similar doses to other patient groups is effective for cognitive enhancement (Ehrenreich et al, 2007a, b) and a single high dose to healthy volunteers enhances memory-dependent hippocampus response after 1 week (Miskowiak et al, 2007b) but not at an earlier time point after administration (Miskowiak et al, 2007c). Depressive symptoms, psychosocial function, and quality of life (QOL) were assessed at week 1 (baseline) and weeks 5, 9, and 14 ( \pm 3 days). Verbal memory was investigated at weeks 1, 9, and 14 using three alternate versions of the Rey Auditory Verbal Learning Test (RAVLT) (original list $\mathrm{AB}, \mathrm{GeAB}$, and $\mathrm{Cr}-\mathrm{AB}$, respectively) administered in a counter-balanced order within each stratum.

The primary outcome was change in HDRS-17 from baseline to week 9. Global assessment of function (GAF) was reported in addition to the primary outcome according to a predetermined dissemination strategy. The secondary outcome measure was remission rate (HDRS-17 score $<8$ ) and tertiary outcome measures were Beck Depression Inventory-21 (BDI-21), World Health Organization QOL BREF (WHOQOL-BREF), and RAVLT (total recall across the five learning trials (I-V), recall following interference (trial VI), recall following a 30 min delay and recognition).

\section{Statistical Analysis}

Comparative analyses between the groups were intentionto-treat (ITT) using last observation carried forward for missing values (primary strategy) as well as per protocol (PP) analyses (not reported). Data were analyzed with repeated-measures analysis of covariance (ANCOVA) with adjustment for stratification variables to minimize effects of any baseline imbalances. For exploratory analysis, we z-transformed and created a mean composite of depression-relevant measures (HDRS-17, GAF, BDI-21, and WHOQOL-BREF) to get a measure of overall depressive syndrome severity. Verbal memory performance was adjusted for HDRS-17 score at week 9 in addition to the stratification variables because of well-documented impact of mood on cognitive function. An exploratory verbal memory composite score was calculated by z-transformation and summation of the four RAVLT subtests. We report differences in means, 95\% CIs, $P$-values, and effect size as reflected by partial $\eta^{2}$ ). The Statistical Package for the Social Sciences was used for the statistical analyses (SPSS, version 19 for IBM).

\section{RESULTS}

\section{Patient Flow and Characteristics}

We randomized the first patient in September 2009 and completed the last patient visit in October 2012 (Figure 1 and Table 1). A total of 98 patients were assessed, of which 58 were excluded. Of the 40 randomized patients, one (EPO) withdrew on the inclusion day (baseline); data were hence collected and analyzed for 39 patients (EPO, $N=18$; saline, $N=21$ ), of whom 34 completed PP (EPO, $N=14$; saline, $N=20$ ). Eight patients displayed marked improvement in the 2 weeks between screening and trial start (baseline) (HDRS-17: mean reduction (SD): 5 (2)) and no longer fulfilled depression severity criteria at this time. These patients were nevertheless included and were equally distributed between the two groups. Of the five patients who did not complete $\mathrm{PP}$, three patients (EPO) discontinued medication after 5-6 infusions because of increased thrombocytes $\left(>4 \times 10^{9} / 1\right)$ but completed all assessments and two patients (one EPO, one placebo) were admitted to the hospital because of acute suicide risk. Groups were well matched in terms of age, gender, and baseline characteristics $(P$-values $>0.12)$. All patients continued their medication as usual (Table 1).

\section{Primary Outcomes}

Results for HDRS-17 and GAF are presented in Table 2. HDRS-17 revealed no effect of EPO over saline at week 9 (mean reduction (95\% CI): EPO, 5.3 (2.0-8.6); saline, 3.5 $(1.6-5.5) ; P=0.32)$ or 14 (mean reduction (95\% CI): EPO, 5.7 (2.7-8.3); saline, $5.2(2.8-7.6) ; P=0.63)$. GAF showed a trend toward enhanced psychosocial function in EPO- $v s$ saline-treated patients at week 9 (mean increase (95\% CI): EPO, 5.1 (1.8-8.3); saline, 1.8 ( -0.9 to 4.6$)$; $P=0.09$ ), which disappeared at follow-up (mean increase (95\% CI): EPO, 4.3 (1.0-7.6); saline: $4.0(0.0-8.1) ; P=0.20)$.

\section{Secondary and Tertiary Outcomes}

Remission rates did not differ between groups at weeks 9 (EPO: $N=4$; saline: $N=2$; Fisher's exact test: $P=0.39$ ) or 14 (EPO: $N=2$; saline: $N=4$; Fisher's exact test: $P=0.67$ ). However, EPO improved BDI-21 (mean reduction (95\% CI): EPO, 9.6 (3.9-15.3); saline, 1.9 ( -1.9 to 5.7$) ; P=0.02$; partial $\eta^{2}=0.14$ ), an effect that was maintained at week 14 (mean reduction (95\% CI): EPO, 7.4 (2.4-12.5); saline, 4.5 (1.37.7); $\left.P=0.04 ; \eta^{2}=0.07\right)$. EPO also improved WHOQOLBREF (mean increase (95\% CI): EPO, 27.5 (11.2-43.8); saline, $-1.2(-12.8$ to 10.4$\left.) ; P=0.01 ; \eta^{2}=0.19\right)$, and this was maintained at follow-up (mean increase (95\% CI): EPO, 28.2 (11.9-44.4); saline, 0.1 ( -11.1 to 11.3$) ; P=0.01$; partial $\left.\eta^{2}=0.12\right)$.

Results for verbal memory are presented in Table 3. EPO enhanced total recall across the five learning trials compared with saline (mean increase (95\% CI): EPO, 6.2 (3.2-9.2); saline: 1.9 ( -0.5 to 4.2$) ; P=0.02$; partial $\left.\eta^{2}=0.16\right)$, and this effect was maintained at follow-up (mean increase (95\% CI): EPO, 7.6 (2.9-12.3); saline: 1.2 ( -1.8 to 4.1$) ; P=0.03$; partial $\left.\eta^{2}=0.11\right)$. EPO also improved recognition memory (mean increase (95\% CI): EPO, 1.7 (0.5-2.9); saline, -0.1 ( -1.0 to $0.8) ; P=0.03$; partial $\left.\eta^{2}=0.15\right)$, which was maintained at week 14 (mean increase (95\% CI): EPO, 1.0 ( -0.4 to 2.4 ); 


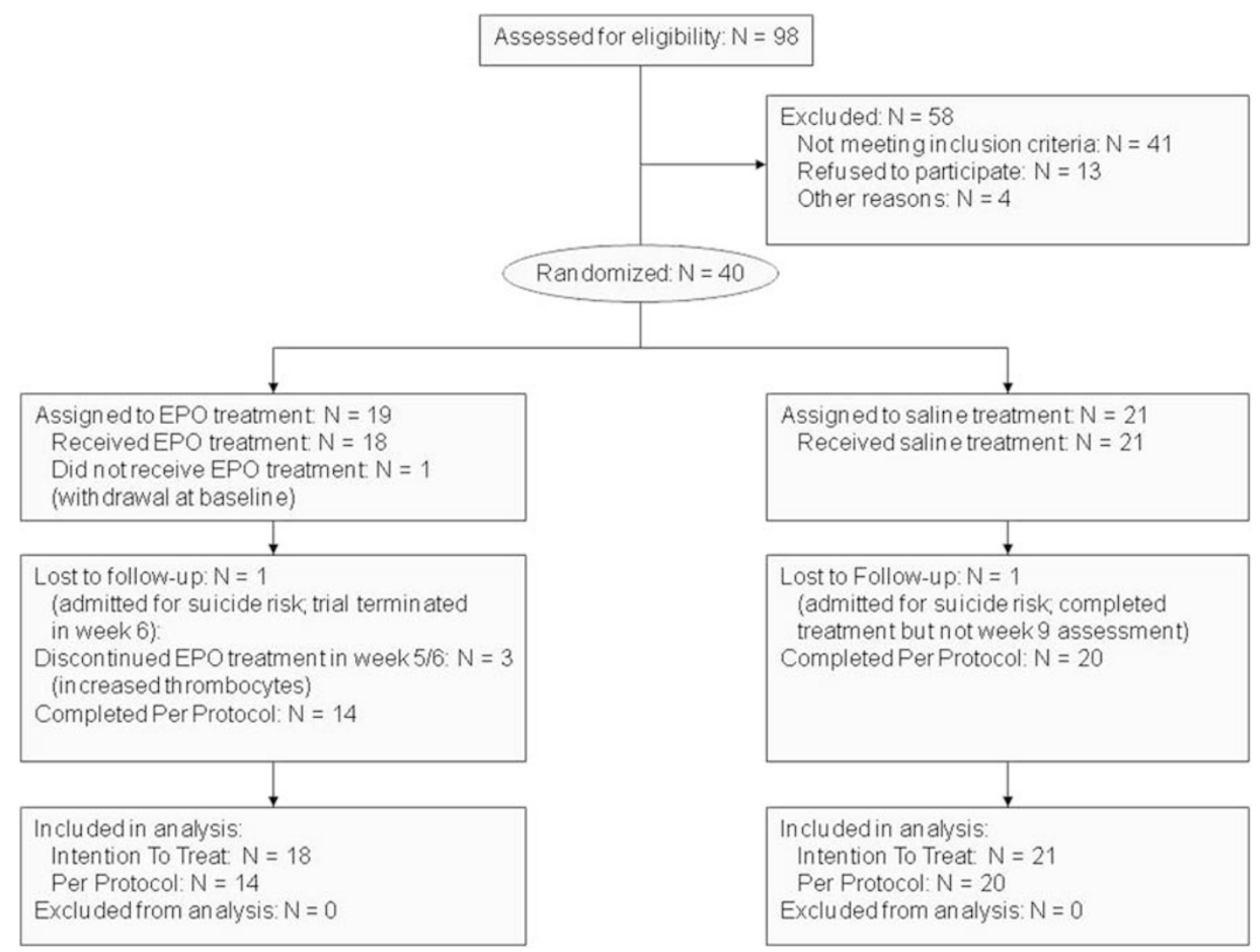

Figure I Patient flow through the phases of the trial.

saline, -0.7 ( -1.6 to 0.3$) ; P=0.04$; partial $\left.\eta^{2}=0.10\right)$. There was a trend toward improvement of recall following interference and delayed recall in EPO vs saline groups (mean increase (95\% CI)—immediate recall: EPO, $1.5(0.2-$ 2.7); saline: $-0.1(-1.3$ to 1.2$) ; P=0.11$; delayed recall: EPO, 1.3 ( -0.3 to 2.9 ); saline: -0.5 ( -1.5 to 0.6$), P=0.08)$, which disappeared at follow-up $(P$-values $>0.16)$.

\section{Exploratory Analyses}

Because of the unexpected mood improvement in eight patients before trial start (at which point they therefore no longer fulfilled the depression severity criteria), we decided to perform an exploratory subgroup analysis excluding these individuals (see the lower part of Table 2). This revealed a beneficial effect of EPO $(N=17)$ over saline $(N=14)$ on the primary outcome, HDRS-17 (mean reduction (95\% CI): EPO, 7.1 (3.9-10.2); saline, 2.8 (0.5-5.0); $P=0.02$; partial $\left.\eta^{2}=0.18\right)$, which was sustained at week 14 (mean reduction (95\% CI): EPO, 7.1 (4.1-10.2); saline, 4.7 (1.7-7.7), $P=0.05$; partial $\left.\eta^{2}=0.09\right)$. In this subgroup, EPO also improved GAF (mean increase (95\% CI): EPO, 6.1 (2.59.8); saline, $1.1(-1.7$ to 3.8$) ; P=0.02$; partial $\left.\eta^{2}=0.19\right)$ and (as in the whole cohort) BDI-21 and QOL (see Table 2), and these effects were maintained at week 14 ( $P$-values $<0.05)$.

Despite the negative primary outcome, the positive signal in the additional depression-relevant measures let us to perform a post hoc exploratory analysis of an overall score of 'depressive syndrome severity', integrating the four depression-relevant outcomes. This revealed improvement in EPO- $(N=18)$ vs saline-treated patients $(N=21)$. This result was obtained both by using the depressive syndrome severity score (mean reduction $(95 \% \mathrm{CI})$ : EPO, $1.2(-0.6$ to $3.0)$; saline, $-1.1(-1.9$ to -0.2$) ; P=0.02$; partial $\eta^{2}=0.15$; weeks $1-14$ : EPO, $0.7(-0.8$ to 2.2$)$; saline, $-0.6(-1.7$ to 0.5$) ; P=0.02$; partial $\left.\eta^{2}=0.09\right)$ (see Table 2$)$ and by calculating percent reduction in depressive syndrome severity based on individual baseline levels (see Figure 2a).

Because of the effects of EPO on total recall and recognition and strong trend thereof on recall following interference and delayed recall, we integrated these four RAVLT measures into one 'verbal memory' composite score. Exploratory analysis of this score showed highly significant longterm effects of EPO over saline. This result was obtained both by using the composite score (mean increase $(95 \%$ CI)-week 1-9: EPO, 1.2 ( -0.1 to 2.5$)$; saline, -0.9 ( -1.8 to 0.6$) ; P=0.003$; partial $\eta^{2}=0.25$; weeks $1-14$ : EPO, 1.2 ( -0.1 to 2.5$)$; saline, $-1.1(-2.0$ to 0.2$) ; P=0.004$; partial $\left.\eta^{2}=0.16\right)$ and by calculating the percent improvement based on individual baseline level (see Figure 2b).

\section{Group-Independent Changes in Outcomes Over Time}

Repeated-measures ANCOVA revealed no placebo response or general improvements in psychosocial function or QOL 
Table I Patient Characteristics

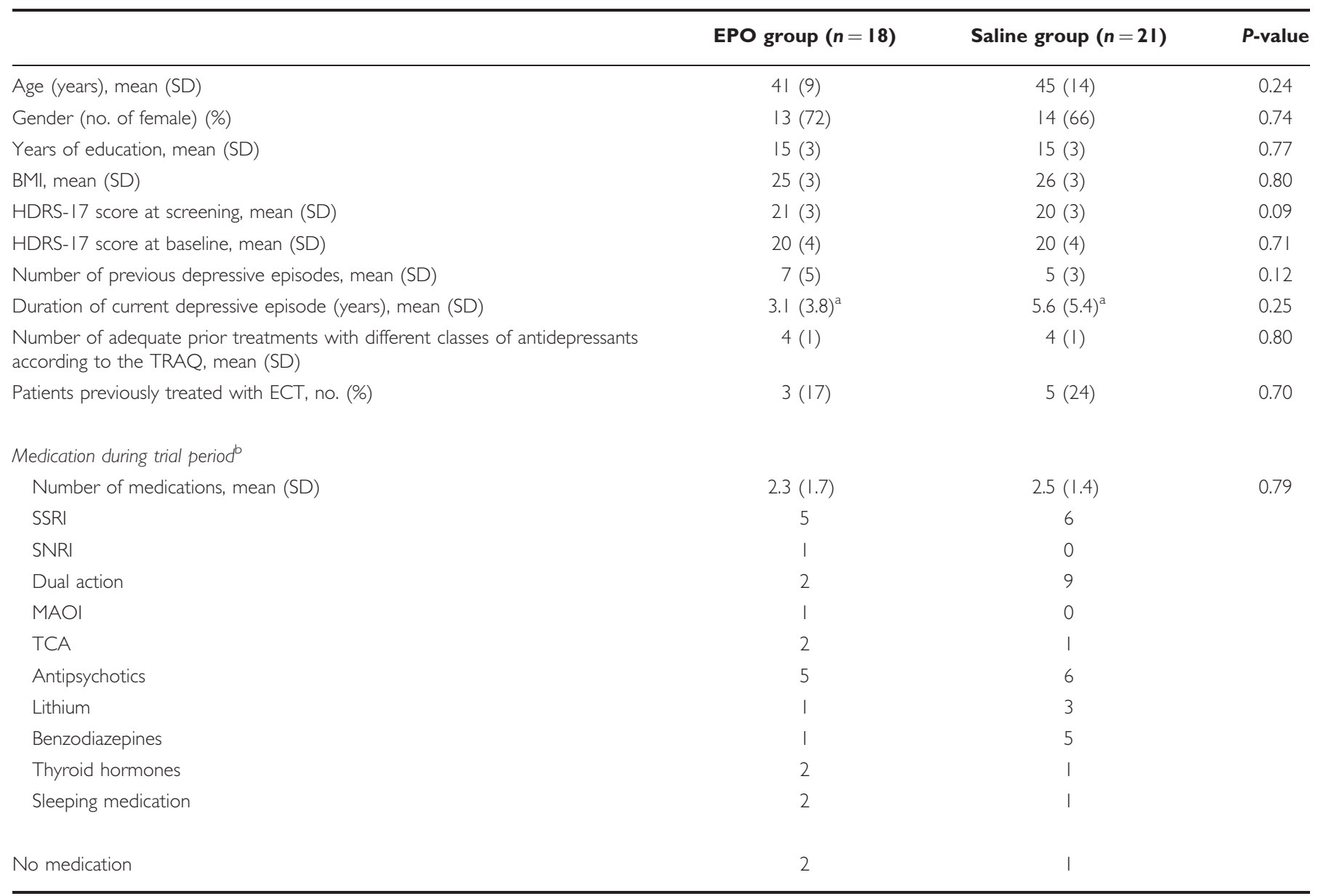

Abbreviations: BMI, body mass index; ECT, electroconvulsive treatment; EPO, erythropoietin; HDRS- I7, Hamilton Depression Rating Scale; MAOI, monoamine oxidase inhibitors; SNRI, selective noradrenaline reuptake inhibitors; SSRI, selective serotonin reuptake inhibitors; TCA, tricyclic antidepressants; I7-TRAQ, Treatment Response to Antidepressants Questionnaire.

${ }^{2}$ Duration of the current episode was only obtained for nine patients (50\%) in the EPO group and 14 patients (67\%) in the saline group.

${ }^{\mathrm{b}} \mathrm{No}$ patients made changes in their medication from weeks I to 9; after week 9, medication change was performed for eight patients (EPO, $N=2 ;$ saline, $N=6$ ).

over time in our patient cohort $(P$-values $>0.35)$. Patients showed no general improvement of RAVLT total recall or recognition $(P$-values $>0.17)$, but there was a learning effect on recall following interference and delayed recall (weeks 1-9: $P$-values $<0.01$; weeks 1-14: $P$-values $<0.02$ ).

\section{Safety Outcomes}

No serious adverse events were identified throughout the trial. Weekly blood pressure measurements demonstrated no systolic or diastolic blood pressure change over time $(P$-values $>0.58)$. Hematological measures are presented in the Supplementary Material. As expected, hemoglobin, erythrocytes, and thrombocytes were increased in EPO $v s$ saline groups $(P$-values <0.003). Three EPO-treated patients had to discontinue treatment after weeks 5-6 because of increased thrombocytes to over $4 \times 10^{9} / \mathrm{l}$. One patient (male) reached the criteria for bloodletting (hematocrit $\geqslant 50 \%$ on two consecutive measurements) after 7 weeks and was bled once $(520 \mathrm{ml})$. Between-group comparisons with $t$-tests showed that hemoglobin, erythrocytes, and thrombocytes were normalized 2 weeks after treatment completion (week 10: $P$-values $>0.05$; week 14: $P$-values $>0.12$ ).

\section{DISCUSSION}

This is the first exploratory study of EPO as a novel treatment for mood symptoms and memory deficits in treatment-resistant depression. Depression severity measured with the HDRS-17, the primary outcome, was not reduced by EPO. Therefore, the study must be declared formally negative. Nevertheless, we did find some indication for antidepressant efficacy of EPO. First, EPO-treated patients showed reduction in BDI ratings of depression and increased QOL compared with those given saline, and these effects persisted until at least 6 weeks after treatment completion. Further, exploratory subgroup analysis excluding eight patients, who were in marked improvement before trial start (baseline) and failed depression severity criteria at this time, revealed improvement of the primary and additional depression-relevant outcomes in EPO vs saline groups, which persisted long term. Finally, because of 
Table 2 Depression Severity (HDRS-17), Psychosocial Function (GAF), Self-Rated Depressive Symptoms (BDI-2I), Quality of Life (WHOQOL-BREF), and Depressive Syndrome Severity (Composite Score for HDRS- I7, GAF, BDI-2I, and WHOQOL-BREF) at Baseline (Week I), Half-Way Through Treatment (Week 5), Upon Treatment Completion (Week 9), and at 6-Week Follow-Up After Treatment Completion (Week 14)

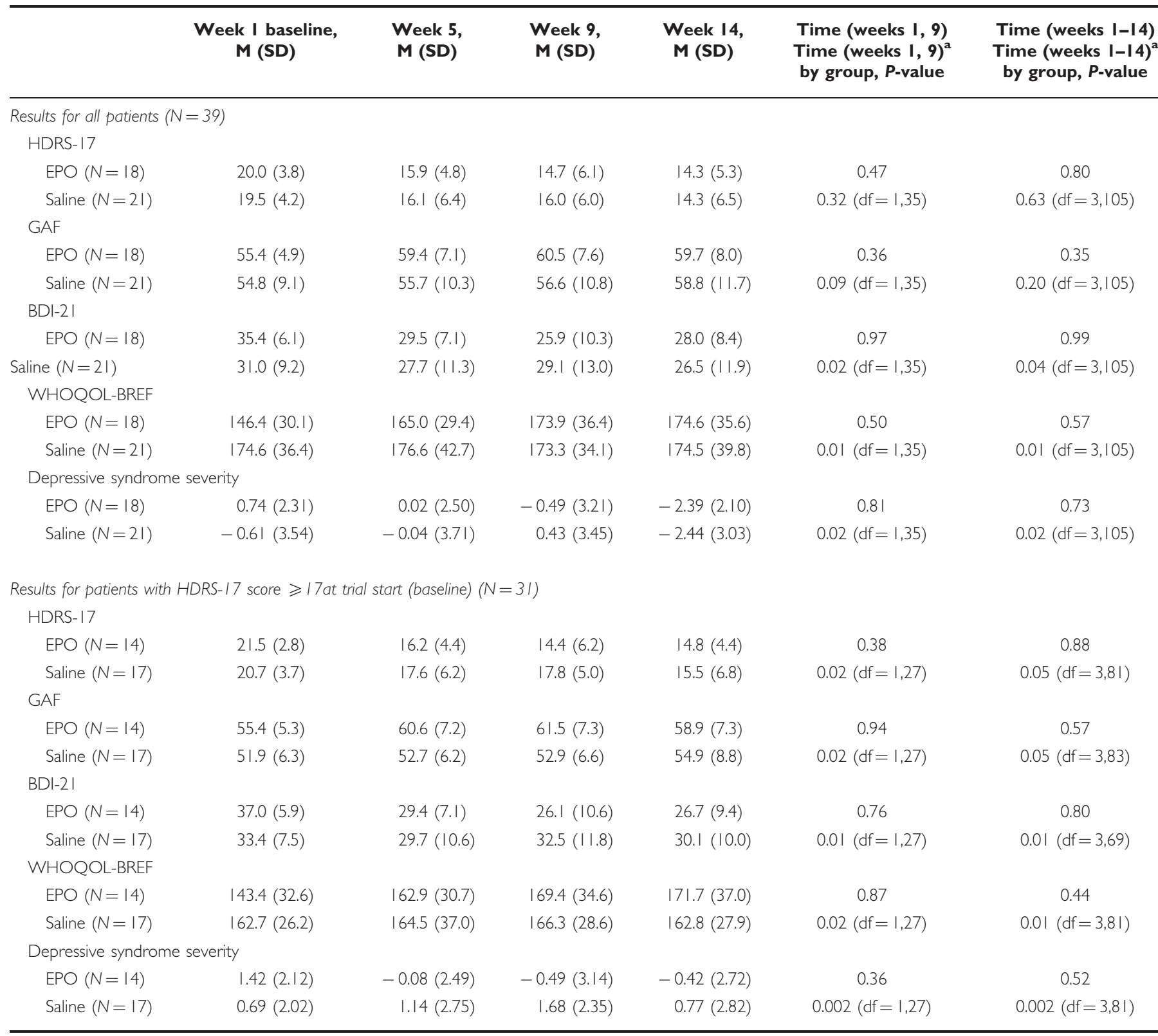

Abbreviations: BDI-2I, Beck Depression Inventory; EPO, erythropoietin; GAF, global assessment of function; HDRS- I7, I7-item Hamilton Depression Rating Scale; M, mean; SD, standard deviation; WHOQOL-BREF, World health Organization Quality of Life BREF questionnaire (total score).

Note: There may be slight differences in these numbers to the 'change scores' reported in the text because of rounding up/down to round numbers.

Covariates for repeated-measures ANCOVA: age and gender.

${ }^{a}$ Degrees of freedom $(\mathrm{df})$ are identical for time and time by group.

positive signal on the additional depression-relevant measures, we performed an exploratory analysis in the complete cohort of 'depression syndrome severity' including depression severity, psychosocial function, and QOL; this exploratory analysis also revealed long-lasting improvement in EPO vs saline groups. This is worth noting given the short EPO treatment schedule relative to patients' depression chronicity and recurrence, and should encou- rage larger-scale clinical studies of EPO in treatmentresistant depression.

EPO produced mood-independent enhancement of verbal memory, which was maintained 6 weeks after treatment completion despite normalization of red blood cell levels. This suggests a mechanism beyond that of red blood cell regulation and is consistent with the actions of EPO on neuroplasticity and neurogenesis. We did not re-examine 
Table 3 Verbal Memory Assessed with the RAVLT at Baseline (Week I), Upon Treatment Completion (Week 9), and at 6-Week FollowUp After Treatment Completion (Week 14)

\begin{tabular}{|c|c|c|c|c|c|}
\hline & $\begin{array}{c}\text { Week I baseline, } \\
\text { M (SD) }\end{array}$ & $\begin{array}{l}\text { Week 9, } \\
\text { M (SD) }\end{array}$ & $\begin{array}{l}\text { Week I4, } \\
\text { M (SD) }\end{array}$ & $\begin{array}{l}\text { Time (weeks I, 9) } \\
\text { Time (weeks I, 9) } \\
\text { by group, P-value }\end{array}$ & $\begin{array}{l}\text { Time (weeks I-I4) } \\
\text { Time (weeks I-I4) } \\
\text { by group, P-value }\end{array}$ \\
\hline \multicolumn{6}{|c|}{ Total recall across the five learning trials (trials $I-V$ ) } \\
\hline $\mathrm{EPO}(N=17)$ & $44.1(10.9)$ & $50.3(9.2)$ & $51.6(9.2)$ & 0.17 & 0.52 \\
\hline Saline $(N=20)$ & $43.5(9.4)$ & $45.4(10.8)$ & $44.7(9.5)$ & $0.02(\mathrm{df}=1,32)$ & $0.03(\mathrm{df}=2,64)$ \\
\hline \multicolumn{6}{|c|}{ Immediate recall following interference (trial VI) } \\
\hline $\mathrm{EPO}(N=17)$ & $8.9(2.8)$ & $10.4(2.4)$ & $10.5(3.0)$ & 0.001 & 0.003 \\
\hline Saline $(N=20)$ & $8.9(3.7)$ & $8.9(3.7)$ & $9.0(3.5)$ & $0.11(\mathrm{df}=1,32)$ & $0.23(\mathrm{df}=2,64)$ \\
\hline \multicolumn{6}{|c|}{ Delayed recall (30 min) } \\
\hline $\mathrm{EPO}(N=17)$ & $8.8(3.2)$ & $10.1(3.5)$ & $10.0(3.0)$ & 0.01 & 0.02 \\
\hline \multicolumn{6}{|l|}{ Recognition } \\
\hline $\mathrm{EPO}(\mathrm{N}=17)$ & I $1.8(2.9)$ & | $3.5(1.6)$ & | $2.8(1.8)$ & 0.69 & 0.92 \\
\hline Saline $(N=19)$ & $12.7(2.4)$ & $12.6(2.1)$ & I2.1 (2.4) & $0.03(\mathrm{df}=\mid, 3 \mathrm{I})$ & $0.04(d f=2,62)$ \\
\hline \multicolumn{6}{|l|}{ Composite score } \\
\hline $\mathrm{EPO}(N=17)$ & $-0.2(3.5)$ & I.0 (3.0) & I.I (3.1) & 0.11 & 0.24 \\
\hline Saline $(N=19)$ & $0.4(3.5)$ & $-0.5(3.4)$ & $-0.7(3.8)$ & $0.003(d f=1,31)$ & $0.004(d f=2,62)$ \\
\hline
\end{tabular}

Abbreviations: EPO, erythropoietin; M, mean; RAVLT, Rey Auditory Verbal Learning Test; SD, standard deviation.

RAVLT data were missing for two patients (one EPO, one saline), hence analyses included: $N=37$ patients; an additional patient (saline) had no baseline recognition data, hence analysis: $N=36$ for this subtest. Composite score: sum of total learning, immediate and delayed recall, and recognition scores. Note that there may be slight differences in these numbers to the 'change scores' reported in the text because of rounding up/down to round numbers.

Note: There may be slight differences in these numbers to the 'change scores' reported in the text because of rounding up/down to round numbers.

Covariates for repeated-measures ANCOVA: age, gender, and depression severity (HDRS-17 score) at week 9.

${ }^{a}$ Degrees of freedom (df) are identical for time and time by group.

memory beyond 6 weeks after treatment completion; however, it is likely that the effects persisted longer-term given evidence for sustained cognitive effects of similar EPO treatment regimens in other patient groups for up to 6 months (Ehrenreich et al, 2007a, b). Given the concomitant upregulation of hematocrit in the present trial, we cannot exclude the possibility that the observed effects of EPO were in part mediated by indirect hematological actions and brain oxygenation. However, several factors speak against this. Studies in other patient populations were able to dissociate between the effects of EPO on cognition and on hematological parameters (Ehrenreich et al, 2007a, b). In keeping with this, we found no correlation between the change in hematocrit and change in HDRS-17 scores $(r(37)=0.19$, $P=0.91)$, depressive syndrome severity $(r(37)=-0.11$, $P=0.51)$ or verbal memory $(r(37)=0.23, P=0.16)$ from baseline to week 9. Perhaps even more convincingly, nonhematopoietic derivatives of EPO such as carbamylated EPO have also been found to produce antidepressant-like effects and cognitive enhancement through central actions in the nervous system (for a review see Miskowiak et al, 2012).

The EPO-associated memory improvement was twice the size of our a priori estimation of a clinically relevant change (Miskowiak et al, 2010). Comparison with norms from an age-matched healthy population with average intelligence (Strauss et al, 2006) showed that EPO improved memory from below to above the level of healthy individuals (see Figure 2b). As memory is an aspect of cognitive function of particular importance for patients' occupational function (Mur et al, 2009), this enduring effect of EPO could enhance patients' functional outcome. It is also tempting to speculate that EPO could be implemented to aid speed of recovery and effectiveness of cognitive behavioral therapy for treatment-resistant depression; in combination these treatments could create synergy between restoration of neuroplasticity, enhanced learning potential, and implementation of goal-directed cognitive and behavioral change.

The trial has a number of limitations. The extensive exclusion criteria may limit the ability to generalize our findings to clinical practice. However, the criteria were inevitable to ensure patient safety in this first trial of its kind, which was of principal importance. Eight patients showed unexpected symptom improvement before trial start. Although these patients were balanced between drug groups, their inclusion may have masked an effect of EPO on the primary outcome. Indeed, exploratory analyses excluding these individuals revealed clear effects of EPO on the primary study outcome. Future trials evaluating the 


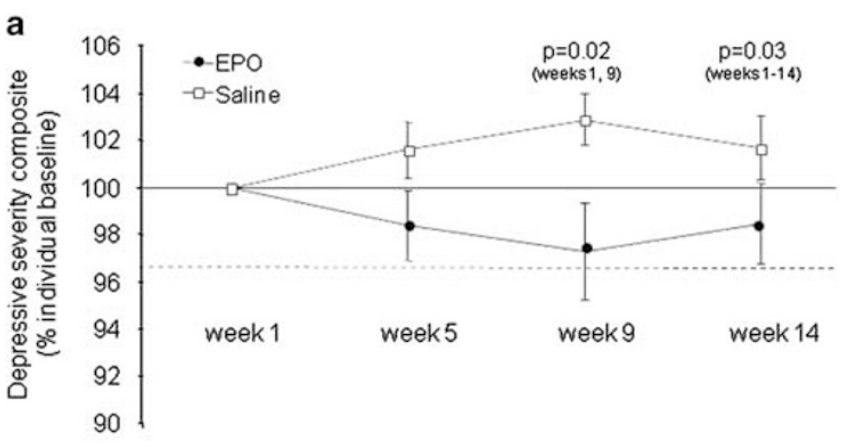

b

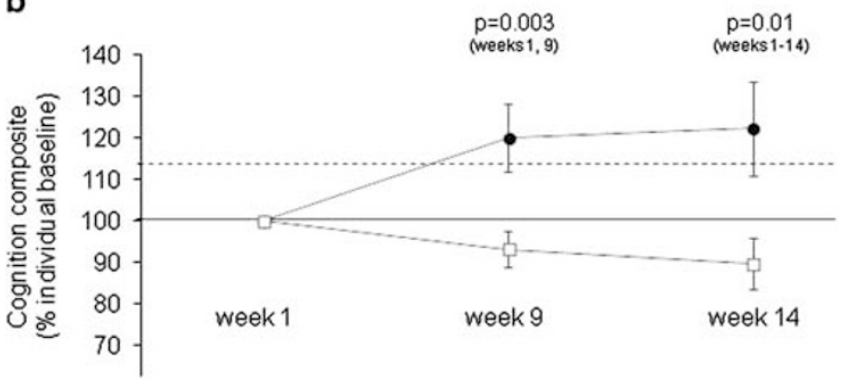

Figure 2 Percent improvement of explorative depression and cognition composite scores from individual baseline. (a) Explorative depression composite. The dotted line denotes the estimated depression composite of healthy individuals within the lower normal range calculated by z-transformation and averaging the cutoffs 17-item Hamilton Depression Rating Scale (HDRS- I7) $=7$, Beck Depression Inventory-2I $(\mathrm{BDI}-2 \mathrm{I})=7$, global assessment of function $(\mathrm{GAF})=70$, and the mean World Health Organization Quality of Life BREF (WHOQOL-BREF) score in the Danish population (Noerholm et al, 2004). (b) Explorative Rey Auditory Verbal Learning Test (RAVLT) composite. This was obtained by summation of the four z-transformed RAVLT measures, addition of 10 to these summed score to obtain overall positive values, and calculation of percent change from individual baseline. P-values indicate the results of the analysis of covariance (ANCOVA) of the mean change from the individual baseline between the drug groups. The dotted line denotes the estimated RAVLT memory composite of healthy, age-matched individuals of average intelligence (Strauss et al, 2006). Mean and SEM are presented.

antidepressant efficacy of EPO may therefore wish to use a run-in phase and include only patients with no spontaneous symptom improvement. The EPO-associated increase in red blood cell levels could confound the interpretation of the effects of EPO as neural in origin. However, converging evidence from animal and human proof-of-concept studies suggest that the ability of EPO to improve cognitive function and produce antidepressant-like effects is mediated by direct actions on neuroplasticity (Miskowiak et al, 2012). In keeping with this, the present effects of EPO on verbal memory and depressive syndrome severity persisted for at least 1 month after red blood cell normalization. In the present trial, we did not screen for or exclude co-morbid axis II disorder as this would have resulted in a subsample of patients who were not representative for the target population of treatment-resistant patients. Nevertheless, assessment of such comorbidity should be performed in future trials with larger patient numbers to allow for explorative subgroup analyses of treatment response in patients with or without personality disorder to assess the potential influence of axis II diagnosis on treatment response. The clinical application of EPO is limited by its hematopoietic actions, which increase the risk of hypertension and blood clotting. However, with careful safety monitoring it is possible that these unwanted blood effects are outweighed by the beneficial actions of EPO in these chronically ill, low-functioning patients. A future solution may be to implement EPO analogs such as carbamylated EPO, which are engineered to have neuroprotective but not hematopoietic actions (Leist et al, 2004). Finally, we are aware of the small groups analyzed that turned out to provide suboptimal power for detection of a clinically relevant difference between groups on the primary outcome. In particular, our original power calculations (Miskowiak et al, 2010) suggested that a sample size of $N=40$ would provide $86 \%$ power to detect a clinically relevant difference of three scores in the change in HDRS-17 between groups, with a standard deviation of three scores at an $\alpha$-level of 5\% (twosided test). However, in our completer sample of $N=39$, the mean difference between groups in the change in HDRS-17 scores was 1.8 with a standard deviation of 5.5 . We therefore performed a post hoc power calculation based on these values, which showed that our sample provided only $67 \%$ power to detect a clinically relevant difference in HDRS-17 scores between groups. Therefore, this suggests that our study may not have been adequately powered to detect a significant effect on our primary outcome measure, although a positive signal was apparent on the additional depressionrelevant outcomes and explorative score of depressive syndrome severity. Based on the exploratory findings, further investigation of EPO to target treatment-resistant depression in a larger patient sample is thus warranted. Indeed, post hoc sample size calculation showed that for detection of a clinically relevant difference in the change in HDRS-17 scores between treatment groups such studies would need a somewhat larger sample size of $N=53$ to obtain adequate power of $80 \%$. Nevertheless, this exploratory study, which is the first of its kind, is convincing when considering that patients had been treated for years without any improvement, and that a treatment period of 8 weeks is very short in such chronic, recurrent condition.

In conclusion, EPO is an interesting compound for addon treatment of mood and memory dysfunction in patients with treatment-resistant depression. Based on the findings of this exploratory study, larger clinical trials of EPO as a treatment for mood symptoms and cognitive deficits in treatment-resistant depression are therefore mandatory.

\section{FUNDING AND DISCLOSURE}

KWM has received consultancy fees from Lundbeck; MV has been a consultant for Eli Lilly, Lundbeck, Servier, and Astra Zeneca; EMC is consultant for Lilly, BMS, Servier, Lundbeck, Astra-Zeneca, and Medilink; JDB reports no potential conflicts of interest; $\mathrm{CJH}$ has received consultancy fees from P1VITAL, Servier, and Eli-Lilly, is a company director of Oxford Psychologists, and has also received grant income from GSK, Lundbeck, Servier, and Astra Zeneca; HE has submitted/holds user patents for EPO in stroke, schizophrenia, and MS; LVK has within the past 3 years been a consultant for Lundbeck, AstraZeneca, and Servier. 


\section{ACKNOWLEDGEMENTS}

We thank the study nurses, Susanne Sander, and Hanne Nikolajsen for their great work with logistical planning and running the trial, cardiologist Steen Pehrson, MD, DMSc, for continuous help with checking ECGs, and physicians at the Clinic for Affective Disorder, and Ulla Knorr, MD, PhD and Astrid Søchting, MD, for their help with patient recruitment and assistance during holiday periods. The study was funded by the Danish Ministry of Science, Innovation, and Higher Education, Novo Nordisk Foundation, Beckett Fonden, and Savværksejer Juhl's Mindefond. Funders had no involvement in the formulation of the hypotheses, data analysis, or in any aspect of preparation of the manuscript and the named researchers were independent from funders.

\section{Authors contribution}

KWM had full access to all the data in the study and takes responsibility for the integrity of the data and the accuracy of the data analysis. KWM, MV, and LVK were responsible for the original proposal. KWM drafted the original protocol and secured funding for the trial and necessary approvals from regulatory bodies. LVK as sponsor and principal investigator had the overall responsibility for the trial. KWM had the responsibility for management of the study, ratings of affective symptoms and training, and supervision of research assistants in cognitive testing. MV and KWM were responsible for patient recruitment. MV was in charge of safety monitoring throughout the trial, including weekly control of blood test results and physical assessments for any potential side effects. MV was also responsible for ordering study medication and ensuring that the cold chain was maintained. HE provided advice on the study design, drug administration, and safety aspects for the original proposal and ongoing safety advice throughout the trial. EMC was involved in recruitment and screening of patients for the study and drug administration. JDB was involved in screening of patients, preparation of study medication during holiday periods, and ratings of affective symptoms in KWM's absence. CJH provided advice on the cognitive test battery for the original proposal. KWM, MV, and LVK were responsible for the statistical analysis plan. $\mathrm{MV}, \mathrm{LVK}, \mathrm{CJH}$, and $\mathrm{HE}$ contributed to the refinement of the trial protocol. KWM was responsible for data collection and analysis in collaboration with LVK. MV and LVK contributed to interpretation of the data. KWM wrote the initial draft of the report. All authors contributed to and approved the final report.

\section{REFERENCES}

Adamcio B, Sargin D, Stradomska A, Medrihan L, Gertler C, Theis $\mathrm{F}$ et al (2008). Erythropoietin enhances hippocampal long-term potentiation and memory. BMC Biol 6: 37.

Brines ML, Ghezzi P, Keenan S, Agnello D, de Lanerolle NC, Cerami C et al (2000). Erythropoietin crosses the blood-brain barrier to protect against experimental brain injury. Proc Natl Acad Sci USA 97: 10526-10531.

Curran HV, Morgan C (2000). Cognitive, dissociative and psychotogenic effects of ketamine in recreational users on the night of drug use and 3 days later. Addiction 95: 575-590.
Danysz W, Wroblewski JT, Costa E (1988). Learning impairment in rats by $N$-methyl-D-aspartate receptor antagonists. Neuropharmacology 27: 653-656.

Ehrenreich H, Fischer B, Norra C, Schellenberger F, Stender N, Stiefel M et al (2007a). Exploring recombinant human erythropoietin in chronic progressive multiple sclerosis. Brain 130: 2577-2588.

Ehrenreich H, Hinze-Selch D, Stawicki S, Aust C, Knolle-Veentjer $\mathrm{S}$, Wilms $\mathrm{S}$ et al (2007b). Improvement of cognitive functions in chronic schizophrenic patients by recombinant human erythropoietin. Mol Psychiatry 12: 206-220.

Furey ML, Drevets WC (2006). Antidepressant efficacy of the antimuscarinic drug scopolamine: a randomized, placebocontrolled clinical trial. Arch Gen Psychiatry 63: 1121-1129.

Girgenti MJ, Hunsberger J, Duman CH, Sathyanesan M, Terwilliger R, Newton SS (2009). Erythropoietin induction by electroconvulsive seizure, gene regulation, and antidepressant-like behavioral effects. Biol Psychiatry 66: 267-274.

Hasselbalch BJ, Knorr U, Kessing LV (2011). Cognitive impairment in the remitted state of unipolar depressive disorder: a systematic review. J Affect Disorder 134: 20-31.

Kästner A, Grube S, El-Kordi A, Stepniak B, Friedrichs H, Sargin D et al (2012). Common variants of the genes encoding erythropoietin and its receptor modulate cognitive performance in schizophrenia. Mod Med 18: 1029-1040.

Leconte C, Bihel E, Lepelletier FX, Bouet V, Saulnier R, Petit E et al (2011). Comparison of the effects of erythropoietin and its carbamylated derivative on behaviour and hippocampal neurogenesis in mice. Neuropharmacology 60: 354-364.

Leist M, Ghezzi P, Grasso G, Bianchi R, Villa P, Fratelli M et al (2004). Derivatives of erythropoietin that are tissue protective but not erythropoietic. Science 305: 239-242.

Manji HK, Quiroz JA, Sporn J, Payne JL, Denicoff K, A Gray N et al (2003). Enhancing neuronal plasticity and cellular resilience to develop novel, improved therapeutics for difficult-to-treat depression. Biol Psychiatry 53: 707-742.

Marti HH, Wenger RH, Rivas LA, Straumann U, Digicaylioglu M, Henn V et al (1996). Erythropoietin gene expression in human, monkey and murine brain. Eur J Neurosci 8: 666-676.

Miskowiak KW, Vinberg M, Harmer CJ, Ehrenreich H, Kessing LV (2012). Erythropoietin: a candidate treatment for mood symptoms and memory dysfunction in depression. Psychopharmacology (Berl) 219: 687-698.

Miskowiak K, O’Sullivan U, Harmer CJ (2007a). Erythropoietin reduces neural and cognitive processing of fear in human models of antidepressant drug action. Biol Psychiatry 62: 1244-1250.

Miskowiak K, O'Sullivan U, Harmer CJ (2007b). Erythropoietin enhances hippocampal response during memory retrieval in humans. J Neurosci 27: 2788-2792.

Miskowiak K, Inkster B, Selvaraj S, Goodwin G, Harmer C (2007c). Erythropoietin has no effect on hippocampal response during memory retrieval 3 days post-administration. Psychopharmacology (Berl) 195: 451-453.

Miskowiak K, Inkster B, O’Sullivan U, Selvaraj S, Goodwin GM, Harmer CJ (2008). Differential effects of erythropoietin on neural and cognitive measures of executive function 3 and 7 days post-administration. Exp Brain Res 184: 313-321.

Miskowiak KW, Vinberg M, Harmer CJ, Ehrenreich H, Knudsen GM, Macoveanu J et al (2010). Effects of erythropoietin on depressive symptoms and neurocognitive deficits in depression and bipolar disorder. Trials 11: 97.

Moussavi S, Chatterji S, Verdes E, Tandon A, Patel V, Ustun B (2007). Depression, chronic diseases, and decrements in health: results from the World Health Surveys. Lancet 370: 851-858.

Mur M, Portella MJ, Martinez-Aran A, Pifarre J, Vieta E (2009). Influence of clinical and neuropsychological variables on the psychosocial and occupational outcome of remitted bipolar patients. Psychopathology 42: 148-156. 
Noerholm V, Groenvold M, Watt T, Bjorner JB, Rasmussen NA, Bech P (2004). Quality of life in the Danish general populationnormative data and validity of WHOQOL-BREF using Rasch and item response theory models. Qual Life Res 13: 531-540.

Posternak MA, Young D, Sheeran T, Chelminski I, Franklin CL, Zimmerman M (2004). Assessing past treatment history: testretest reliability of the Treatment Response to Antidepressant Questionnaire. J Nerv Ment Dis 192: 95-102.

Rush AJ, Trivedi MH, Wisniewski SR, Nierenberg AA, Stewart JW, Warden D et al (2006). Acute and longer-term outcomes in depressed outpatients requiring one or several treatment steps: a STAR ${ }^{\star D}$ report. Am J Psychiatry 163: 1905-1917.

Sackeim HA (2001). The definition and meaning of treatmentresistant depression. J Clin Psychiatry 62: 10-17.

Sargin D, Friedrichs H, El-Kordi A, Ehrenreich H (2010). Erythropoietin as neuroprotective and neuroregenerative treatment strategy: comprehensive overview of 12 years of preclinical and clinical research. Best Pract Res Clin Anaesthesiol 24: 573-594.

Schmidt M (2004). Rey Auditory Verbal Learning Test-A Handbook. Western Psychological Services: Los Angeles, LA, USA.

Siren AL, Fasshauer T, Bartels C, Ehrenreich H (2009). Therapeutic potential of erythropoietin and its structural or functional variants in the nervous system. Neurotherapeutics 6: 108-127.

Strauss E, Sherman EMS, Spreen O (2006). A Compendium of Neuropsychological Tests. Administration, Norms, and Commentary. Oxford University Press: New York, NY, USA.

Zarate CA Jr., Mathews D, Ibrahim L, Chaves JF, Marquardt C, Ukoh I et al (2006). A randomized trial of an N-methyl-Daspartate antagonist in treatment-resistant major depression. Arch Gen Psychiatry 63: 856-864.

Supplementary Information accompanies the paper on the Neuropsychopharmacology website (http://www.nature.com/npp) 